\title{
CLINICAL AND MICROBIOLOGICAL EVALUATION OF THE EFFECT OF HEAT KILLED LACTOBACILLUS ACIDOPHILUS AND GARLIC EXTRACT ON CANDIDA ALBICANS IN A GROUP OF ELDERLY DENTURE WEARERS
}

\author{
Abdel Hamed B.M.* and Eman Alaa**
}

\begin{abstract}
Continuous denture wearing, lack of adequate hygiene and infection with Candida albicans (C. albicans) are among the etiological factors of Denture stomatitis (DS). The management of DS ranges from the implementation of oral hygiene measures and removal of predisposing factors to the use of antifungals, depending on the severity of inflammation.

Aim of the study: This study aimed to evaluate the short-term effect of heat killed lactobacillus acidophilus - Lacteol Fort ${ }^{\circledR}(\mathrm{LF})$ - as a probiotic and garlic extract -Tomex Plus ${ }^{\circledR}(\mathrm{TP})$ - as a prebiotic on salivary C. albicans counts and biofilm-forming ability, in elderly denture wearers with DS.

Methods: This was a single-center, single-blind, randomized pilot clinical trial. 27 complete denture wearers were randomly allocated into 3 groups; group I (control), group II (LF) and group III (TP). Each group was clinically assessed for DS and microbiologically evaluated for salivary C. albicans counts at baseline and after 15 days. The biofilm-forming ability of salivary C. albicans was assessed, as well, for each group at the end of the experimental period.
\end{abstract}

Results: Clinical assessment revealed a reduction in the total erythematous area dimension from $3.00 \pm 0.40 \mathrm{~mm}$ for group II (LF) and from $3.20 \pm 0.54 \mathrm{~mm}$ for group III (TP) to $\leq 2 \mathrm{~mm}$ for either group after treatment. Microbiological evaluation showed that $\mathrm{C}$. albicans counts and biofilm-forming ability were significantly lowered in groups II (LF) and III (TP).

Conclusion: LF and TP were both found to be effective in reducing DS associated inflammation, C. albicans counts and biofilm-forming ability.

KEYWORDS: Denture stomatitis, prebiotic, probiotic, Candida albicans, biofilm

* Lecturer, Department of Removable Prosthodontics, Faculty of Oral and Dental Medicine, Future University in Egypt, Cairo, Egypt.

** Lecturer, Department of Pediatric Dentistry and Dental Public Health, Faculty of Oral and Dental Medicine, Future University in Egypt, Cairo, Egypt. 


\section{INTRODUCTION}

Candida albicans (C. albicans) is a dimorphic fungus that is normally present in small numbers in the oral flora of humans ${ }^{[1,2]}$. It may exist both as a commensal as well as an opportunistic pathogen ${ }^{[3]}$. The ability of $\mathrm{C}$. albicans to transition from commensal to pathogen is largely due to its ability to change from yeast to hyphal forms, a key virulence factor of this species. This transition is mediated by a rise in $\mathrm{pH}^{[4]}$.C. albicans can catabolize proteins like keratin, releasing ammonia with a consequent rise in $\mathrm{pH}$, favoring hyphal morphology ${ }^{[5]}$.Ultrastructurally, the morphological shift of C. albicans to the hyphal form is mediated through the polarization of the actin cytoskeleton via sequential activation of cyclin and cyclin-dependent $\operatorname{kinase}^{[1,2,6]}$.

The virulence factors of C. albicans also include cellular glycoproteins, such as glucose and mannose, which facilitate adherence to surfaces and formation of biofilms ${ }^{[1]}$. Furthermore, C. albicans possess cellular polysaccharides which are able to repress host defense mechanisms as they inhibit phagocytosis and induce suppressor Tlymphocytes ${ }^{[4,}$ 7]. C. albicans present in biofilms exhibit slower rates of growth yet a superior resistance to antimicrobials and mechanical removal than do planktonic cells ${ }^{[8]}$. A microbial species may be able to construct a biofilm solely, however, the in vivo scenario usually involves an array of bacterial and fungal species ${ }^{[1]}$.

Biofilm formation and C. albicans adherence to denture bases is thought to occur in a two-stage process ${ }^{[7]}$. The primary stage is nonspecific and reversible, while the second stage is via specific intermolecular interactions ${ }^{[1,3]}$. Factors enhancing the adherence of $\mathrm{C}$. albicans include surface roughness, the presence of other adherent microorganisms, and the consumption of a carbohydraterich $\operatorname{diet}^{[9]}$.

Removable appliances are common devices to replace, retain or move teeth in children as well as the elderly ${ }^{[10,11]}$. Those who use a dental prosthesis (orthodontic appliances, removable or complete prosthesis) are susceptible to denture stomatitis associated with C. albicans ${ }^{[12]}$. Polymethylmethacrylates (PMMA) are commonly used materials for denture construction, owing to their simple preparation, stability in the oral environment, esthetically acceptable color and affordable price ${ }^{[9,13]}$. Nevertheless, PMMA absorbs water with consequent polymeric breakdown, which adds to surface crazing and irregularities on the denture fitting surface resulting in microorganism entrapment $^{[13]}$. The denture fitting surface facilitates ammonia production and a subsequent rise in $\mathrm{pH}$ through the degradation of mucous membrane proteins. This rise in $\mathrm{pH}$ along with the warm temperature of the oral cavity promote hyphae formation and consequently the development of denture stomatitis ${ }^{[5,14,15]}$. Newton (1962) classified denture stomatitis (DS) into three main types, type 1, with focal areas of inflammation, type 2: wide spread erythema and type 3: with papillary hyperplasia ${ }^{[2]}$.

DSis one of the most reported denture-related lesions. Moreover, recently its` incidence has been rising, with the emergence of acquired multiple drugresistant strains of C. albicans ${ }^{[16]}$.Like other yeasts, C. albicans can develop evolutionarily mechanisms that lower their susceptibility towards a given drug through the acquisition of different mutations ${ }^{[17-19]}$. These mutations directly or indirectly interfere with the binding of a drug to its targets by inducing gene expression changes that reduce drug susceptibility. In this regard, an enhanced capacity to form biofilms can result in the acquisition of resistance, as these structures promote yeast survival upon exposure to drugs ${ }^{[3,17-19]}$. Research has been focused lately on the development of other therapeutic substitutes for candidiasis management including probiotics, prebiotics, antifungal peptides, sera with monoclonal or polyclonal antibodies or combinations of cytokines ${ }^{[16]}$. 
Health awareness coupled with the fast pace of the modern-day life made "Nutraceutical and functional food (NFF) substances" a familiar component of many daily dietary plans. The word nutraceutical originated from the merging of the words"nutrition" and "pharmaceutical"[20-23]. Nutraceutical denotes unaltered or processed natural compounds that impart health benefits and are generally marketed as food supplements. Probiotics and prebiotics are considered as Nutraceutical/functional foods due to their health benefits ${ }^{[20,21,24]}$.In the year 2002, Food and Agriculture Organization of the United Nations (FAO) defined probiotics as being "live strains of strictly selected microorganisms which, when administered in adequate amounts, confer a health benefit on the host". In 2013, the definition was acknowledged also, by the International Scientific Association for Probiotics and Prebiotics (ISAPP). Prebiotics characterization, however, has been modified for years. In 2007, FAO/WHO experts, finally defined prebiotics as "a nonviable food constituent that confers a health advantage on the host linked to the microbiota modulation"[21-23].

To date, the precise mode of action of probiotics has not been elucidated ${ }^{[24,25]}$. The simplest benefit that research suggests is the competition for nutrients and adhesion sites on the epithelium ${ }^{[26]}$. Another suggested mechanism is through the improvement of epithelial barrier function, by some strains of Lactobacillus spp., through upregulation of IgA synthesis and mucus production ${ }^{[25,26]}$.

Moreover, it has been reported that probiotics control the Nuclear Factor Kappa-Light-ChainEnhancer of activated B cells (NF-kB) pathway ${ }^{[26]}$. They also modulate proteoglycan recognition proteins and toll-like receptors on mucosal cells of the gastrointestinal tract (GIT). The latter subsequently resulting in the activation of T-helper and dendritic cells ${ }^{[21,26,27]}$. Furthermore, probiotics are able to produce antimicrobial compounds like bacteriocins which antagonize the growth of pathogenic microorganisms ${ }^{[21,26]}$.
Lacteol fort (LF) is a commercially available probiotic. It should be noted though that it diverges from the strict description of probiotics, as it is made up of heat-killed micro-organisms and not viable ones. The efficacy of LF in promoting GIT health and in controlling diarrhea has been reported by several researchers over the past decade ${ }^{[28-31]}$. However, its` effect on DS hasnot been reported before in literature, to the best of our knowledge. LF comprises the "probiotic" microorganism, Lactobacillus acidophilus LB Strain. Lactobacillus acidophilus strain is an integral part of the resident human microflora. In vitro and in vivo studies have repeatedly demonstrated this micro-organism`s probiotic activity through the secretion antimicrobial substances and enhancement of the immune response against pathogens ${ }^{[29,32,33]}$. Probiotics were also proved to lessen the risk of hyposalivation and the feeling of dry mouth in elderly patients ${ }^{[34]}$.

Prebiotics, on the other hand, are short-chain carbohydrates that are non-digestible in the upper GIT. Prebiotics are also capable of preferentially enhancing the growth of beneficial bacteria especially, Bifidobacterium and Lactobacillus while retarding that of pathogenic bacteria ${ }^{\text {[21, }}$ 22]. Other benefits that have been described in literature regarding prebiotics are the anticancer effects (colon cancer) and their ability to increase the bioavailability of $\mathrm{Fe} \& \mathrm{Ca}$ and osteoporosis management, especially in children ${ }^{[22,35]}$.

Commonly consumed prebiotics include garlic, onion, asparagus, whole wheat flour, banana, raw wheat bran, raw leek and raw chicory roots. Garlic`s biological activity is attributed to Allicin which gives garlic it's characteristic smell. When garlic is cut or crushed, the enzyme alliinase changes alliin into allicin ${ }^{[36]}$.

Allicin(diallyl thiosulfinate) possess a documented anti-fungal, antiviral, anti-parasitic and anti-bacterial activity ${ }^{[35,37]}$. It has been reported to exhibit a broad-spectrum antimicrobial activity, even against multidrug-resistant bacteria ${ }^{[38-40]}$. 
Allicin can cross cell membranes and combine with sulfur-containing molecular groups in proteins. Consequently, there is oxidation of glutathione with the induction of microbial apoptosis ${ }^{[37,39,41]}$.

Micro-array tests proved that allicin impaired the expression of genes coding for enzymes of amino acid metabolism and iron-uptake [40]. These findings may be explained by the action of allicin on some transcription factors (YAP1, SKN7), which control oxidative stress response in yeast ${ }^{[40,42,43]}$. In addition, allicin appears to stimulate cellular immunity, which gives it an advantage over conventional antifungal drugs ${ }^{[39]}$. The bioactivity and benefits of allicin, justifiably, gave it the title " The heart of garlic"[41].

Tomex plus (TP) is an Egyptia nmade, commercially available enteric-coated tablet of dried garlic powder extract with allicin. Allicin is considered to be a potent prebiotic ${ }^{[20,37,39]}$ that is sold under different brand names. Many brands of garlic are manufactured as enteric-coated tablets to prevent its disintegration in the stomach. The enzyme alliinase - required for allicin release from garlicis inactive at $\mathrm{pH} 3.5$ or lower ${ }^{[36,37,40]}$.Additionally, besides being rich in allicin, TP also contains many essentials micronutrients, like vitamin $\mathrm{A}, \mathrm{C}$ and $\mathrm{E}$ and zinc. Jointly, these micronutrients play a pivotal role in maintaining the functional integrity of mucosal cells, collagen and Lymphocytes and protect cell membranes from damage caused by free radicals, as well ${ }^{[44]}$. TP as a prebiotic and its effect on DS has not been evaluated or reported in literature, to date.

LF and TP are commercially available and cheap. Their probiotic and prebiotic components are assumed to be able to act against a wide range of pathogens and to be immunomodulatory. The above-mentioned characteristics make them ideal candidates for assessment as potential antifungal agents. The present study was designed to assess the effect of LF and TP on salivary C. albicans counts and biofilm-forming ability, in elderly denture wearers, which has not been reported before in literature, to the best of our knowledge.

\section{AIM OF THE STUDY}

This study aimed to evaluate the short-term effect of Lacteol fort (LF) as a probiotic and Tomex plus (TP) as prebiotic on salivary C. albicans counts and biofilm-forming ability, in a group of elderly denture wearers.

\section{MATERIALS AND METHODS}

\section{a) Study design and participants}

This was a single-center, single-blind, randomized pilot clinical trial. The trial took place at the dental hospital of the Faculty of Oral and Dental Medicine (FODM), Future University in Egypt (FUE), Cairo, Egypt.

Through the period from September 2019 to January 2020, 30 complete denture wearers attending the out-patient clinics of the dental hospital of the FODM, FUE, were evaluated for this study. All patients were clinically examined and were asked to fill-out a detailed questionnaire for this study. The questionnaire contained information about; personal data, medical history, how long they have worn the prostheses, last modification to the prosthesis to make sure they had not performed any modification to their dentures or dietary habits over the past 6 months. Patients were considered ineligible if they had any of the exclusion criteria which comprised; inability to perform or understand the experimental procedures, use of antibiotics, immunosuppressives, antifungals and/or antiseptic mouth rinses in the previous 6 months, report of consumption of probiotics over the past 6 months, had severe GI disorders, heart disease, HIV and/or AIDS.

From the 30 evaluated denture wearers, 3 were not considered eligible, as two took antibiotics over the past couple of months and one had had a cardiac 
infarction four months earlier. Twenty-seven patients met the eligibility criteria. The clinical examination and DS classification were performed by two independent certified prosthodontists (to confirm the diagnosis and avoid bias), according to Pattanaik, Vikas et al. 2010 and Martins and de Lacerda Gontijo 2017, [10, 45].From the twentyseven patients included in the study 17 (62.9\%) were male and $10(37.1 \%)$ were female. The average age of the included patients was \pm 61.8 . Criteria for discontinuing intervention were allergies or worsening of the symptoms.

The present study has been performed in accordance with "The Code of Ethics of the World Medical Association" (Declaration of Helsinki) for experiments involving humans ${ }^{[46]}$.All participants were fully informed about the study and signed a detailed informed consent. The experimental design and study protocol described earlier, were approved by the ethical committee of the FODM, FUE (\#FUE. REC-(1)/2020).

\section{b) Grouping, Randomization, allocation conceal- ment and blinding}

\section{Grouping}

The 27 patients included in the study were randomly allocated into one of three groups.

Group I (C): Control (basic standard management only).

Group II (LF): Receiving LF (along with the basic standard management).

Group III (TP): Receiving TP (along with the basic standard management).

Patients in all three groups were instructed to follow basic standards for the management of DS type 1 according to Lombardi and BudtzJørgensen $1993^{[47]}$ and Gade, Pawar et al. 2015 ${ }^{[11]}$, throughout the experimental period. Basic standard management included the following; patients were instructed to maintain good oral hygiene, rinse after meals (3 times daily) with a benzydamine containing mouth wash and stop denture wearing through-out the night. Patients were also directed to remove dentures for as long as possible and to brush their dentures with warm water and immerse them for 15-30 min. twice weekly in a solution of white vinegar (diluted 1:20) $)^{[48]}$.

\section{Lacteal fort ${ }^{\circledR}$, Rameda, Egypt, under license} from ADARE pharmaceuticals (probiotic): each sachet contains Lactobacillus acidophilus LB Strain, 10 billion (Lyophilized killed microbial bodies), Neutralized and lyophilized culture medium 160mg, Excipients: Lactose monohydrate, calcium carbonate, silicic acid, banana-orange flavor, saccharose, q.s.800mg. The allocated patients received a 15-day supply of sachets with the labelling on the sachet of the drug obscured using a non-erasable marker. The dose indicated by the manufacturer for adults was two sachets for the first dose, then 1 sachet twice daily thereafter. The patients were instructed to rinse with the dissolved sachet and keep the fluid in the oral cavity for a minimum of 30 seconds before swallowing.

Tomex Plus®, Sekem, Egypt (prebiotic): each bottle contains 50 odorless garlic entericcoated tablets. $300 \mathrm{mg}$ dried garlic powder tablets with concentrated allicin, Lycopene, Monohydrate Selenium Dioxide, Vitamin A, Vitamin C, Vitamin $\mathrm{E}$ and Zinc Sulphate as active ingredients. A packet with a 15-day supply of tablets was given to the patients. The prescribed dose was according to manufacturer recommendations (three tablets per day).

2. Randomization: Computer-generated randomization was done to randomly assign patients to group I, II or III, using a Microsoft office excel 2007 sheet. This was performed by the prosthodontics clinic secretary with the help of the head of the floor nursing staff, the authors of this study took no part in the randomization process. 
3. Allocation concealment: The codes for allocation were written on a piece of opaque white paper by the secretary of the prosthodontic clinic and the head of the nursing staff. The code was then folded and kept securely at the clinics` secretary office.

4. Blinding: This is a single-blind pilot clinical trial as blinding of the operator (a resident in the prosthodontic department, responsible for taking the drugs from department and giving them to the allocated patients) was not be possible due to the different forms of the tested material (lacteol fort -LF- probiotic- is available in powder form in sachets while, Tomex plus -TP- prebioticis in tablet form). Patients were blinded to the treatment they were receiving. The instructions for taking the allocated treatment were written beforehand by the authors of this study and kept with the drugs, in the drug cabinet in the prosthodontics clinic. Each drug was placed in a separate locked drawer that was labelled with group name on the outside, the keys to the drawers were kept with the head of the nursing staff.

5. Saliva collection: Whole saliva was collected and assessed for all groups at baseline (confirming the presence and counts of $\mathrm{C}$. albicans) and at the termination of the experiment after 15 days.

Salivary samples were collected a day after clinical examination. The collection was made by the authors of this study between 8 to 11 am and subjects were instructed not to eat for two hours prior to the appointment ${ }^{[49]}$. Saliva was collected using 'Spitting Method' for 5 minutes by repeatedly spitting in $50 \mathrm{~mL}$ sterile tubes every 60 seconds $^{[50]}$. After saliva collection, all samples were stored immediately at $4^{\circ} \mathrm{C}$ on BHI broth and sent to the microbiology laboratory where they were cultured within 2 hours. c) Tests

\section{Clinical evaluation}

The erythematous areas were visually counted and dimensions recorded in $\mathrm{mm}$ using a piece of graph paper by two independent prosthodontists and the average was calculated to be used in this study. The total dimensions of erythematous areas measured by each prosthodontist for each patient were summed up, and the mean was recorded at baseline and after 15 days of treatment.

\section{Microbiological evaluation}

Using a sterile loop, $10 \mu$ lof each collected sample was cultured on sabouraud dextrose agar and was incubated at $37 \%$ for 24 hours.

\section{Candida colony forming units}

At baseline, a salivary sample was obtained from each participant and the infection level of Candida sp. was evaluated on a sabouraud dextrose agar plate. This was repeated at the end of the experimental period. The colonies forming on the plates were counted.

\section{Biofilm-forming ability (biofilm growing, staining and quantifying)}

A single colony of C. albicans from sabouraud dextrose agar plate was collected and suspended in sterile BHI broth at $37^{\circ} \mathrm{C}$ overnight for each group. It was then diluted into 1:100 fresh medium then $100 \mu \mathrm{l}$ were added per well in a 96 well dish. For quantitative assays, 8 replicate wells for each treatment were used and finally the microtiter plate was incubated for 24 hours at $37^{\circ} \mathrm{C}$.

After incubation, the cells were dumped out by turning the plate over and shaking out the excess liquid. Then gently the microtiter plate was submerged in a small tub of water then the excess water was shaken out. A125 $\mu \mathrm{l}$ of a $0.1 \%$ solution of crystal violet (CV) in water was added to each well of the microtiter plate. The microtiter plate was then incubated at room temperature for 10-15 min., then 
rinsed 3-4 times with water, by submerging in a water tub. To each well of the microtiter plate, a $125 \mu \mathrm{l}$ of $30 \%$ acetic acid in water was added, to solubilize the $\mathrm{CV}$. Again, the microtiter plate was incubated at room temperature for 10-15 $\mathrm{min}$. To a new flat-bottom microtiter plate, $125 \mu \mathrm{l}$ of the solubilized CV was transferred to quantify absorbance. Absorbance was quantified in a plate reader at $550 \mathrm{~nm}$, using $30 \%$ acetic acid in water as the blank ${ }^{[51]}$.

\section{Data analysis}

The collected data were tabulated using Microsoft Excel (Microsoft Office 2007), through the course of the study. After the completion of the study, the data from the excel sheets were tabulated using the Statistical Package for Social Science (SPSS 15.0) Software. The mean values and standard errors were calculated. Graphs were performed using Microsoft PowerPoint software (Microsoft Office 2007). Differences in the characteristics between the two groups were assessed by one-way ANOVA and Student's t-test. The results were considered significant at $\mathrm{p} \leq 0.05$.

\section{RESULTS}

\section{Clinical evaluation}

Patients of all three groups in the study had a maximum of 3 erythematous areas that measured between 1 to $2 \mathrm{~mm}$ in greatest dimension. By day 15 , groups II and III had exhibited a reduction in the dimensions of the erythematous areas $(\leq 2 \mathrm{~mm})$. While, for group I the mean reduction in dimensions oferythematous areas was only $3 \%$, by day 15 .

\section{C. albicans colonies (infection levels) and bio- film-forming ability}

Using One-way ANOVA and student t-test, it was found that there was no significant differences between the groups at baseline $((\mathrm{p}>0.05)$. At the end of the experimental period, it was found that there was a statistically significant difference among the studied groups $(\mathrm{p}=0.000)$. Student t-test revealed that difference between group I and either group II or III was highly significant $(\mathrm{p}=0.000)$ but the difference between group II and III was statistically insignificant $(\mathrm{p}=.778)$.

As for the Biofilm quantification assay, Oneway ANOVA showed that there was a significant difference among the studied groups and student t-test revealed that there was a significant difference between group I and either groups II or III ( $p \leq$ $0.001)$ and there was a non-significant difference between groups II and III ( $\mathrm{p}>0.05)$.

TABLE 1: Descriptive analysis of the C. albicans counts (infection level) among the studied groups

\begin{tabular}{|c|c|c|c|c|}
\hline \multicolumn{2}{|c|}{ Mean \pm SD } & $\begin{array}{c}\text { Total Erythematous } \\
\text { Area dimension in mm }\end{array}$ & C. albicans counts & $\begin{array}{c}\text { C. albicans } \\
\text { Biofilm-forming ability }\end{array}$ \\
\hline \multirow{2}{*}{ At baseline } & Group I & $3.11 \pm 0.64$ & $49.00 \pm 3.87$ & - \\
\cline { 2 - 5 } & Group II & $3.00 \pm 0.40$ & $48.00 \pm 3.202$ & - \\
\cline { 2 - 5 } & Group III & $3.20 \pm 0.54$ & $48.78 \pm 2.99$ & $5.00 \pm 0.43$ \\
\hline \multirow{2}{*}{$\begin{array}{c}\text { At the end of } \\
\text { experimental } \\
\text { period }\end{array}$} & Group I & $2.69 \pm 0.43$ & $42.00 \pm 4.272$ & $0.26 \pm 0.07$ \\
\cline { 2 - 5 } & Group II & $\leq 2$ & $1.89 \pm 0.928$ & $0.31 \pm 0.08$ \\
\cline { 2 - 5 } & Group III & $\leq 2$ & $3.33 \pm 1.14$ & \\
\hline
\end{tabular}




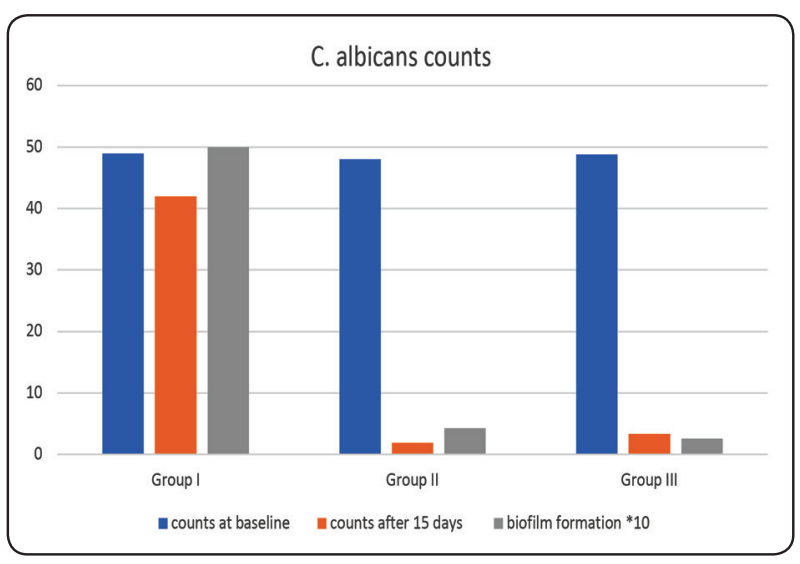

Fig. (1) Column chart showing the mean C.albicans counts and biofilm forming ability in the studied groups

\section{DISCUSSION}

The incidence of $\mathrm{C}$. albicans infection and resistance to treatment has increased over the past couple of decades. This increase is causing alarm among healthcare professionals. This increasehas been attributed to the longer life span of the population besides the overuse of broad-spectrum antimicrobials, immune-suppressive and cytotoxic $\operatorname{drugs} s^{[2,5,7]}$.

Longer life expectancies led to an increase in the number of people requiring removable dentures ${ }^{[11]}$. Denture wearers are especially susceptible to C. albicans infection due to age-associated immune system impairment, together with the irregular denture fitting surface providing an ideal incubator for a biofilm with a substantial microbial population. Most denture wearers are also characteristically older adults who -understandably- lack sufficient physical dexterity to effectively cleanse their appliances $^{[3]}$.

The majority of denture wearers are afflicted with DS during their course of life. The etiology of DS is multifactorial. It has been associated with continuous and long-term use of dentures and poor oral and denture hygiene habits ${ }^{[52]}$.Poor hygiene facilitates colonization of PMMA denture base material by C.albicans, primarily by means of biofilm formation and morphological switching ${ }^{[1,9,13]}$.
The treatment of DS is complex because of its' multifactorial etiology. Management of denture stomatitis includes good oral hygiene, improvement in denture cleansing procedures and discontinuation of nocturnal denture wearing. Topical or systemic antifungal agents may be prescribed, as well, if necessary ${ }^{[4,11,18,53,54]}$. However, antifungals have some drawbacks, from the evolution of treatmentresistant $\mathrm{C}$. albicans strains to the lack of patient compliance due to the burning sensation on topical antifungal application ${ }^{[19,54,55]}$. Systemic antifungals have even more side-effects involving diarrhea, vomiting, hepatotoxicity and cardiac problems which are particularly serious in older patients ${ }^{[18,55]}$. Moreover, antifungals are effective against yeasts alone, while the biofilm on the denture surface is a complex community of yeasts, as well as, bacteria ${ }^{[55]}$.

Collectively, these reasons called for the development of a more effective treatment with fewer side effects. The current study was designed to evaluate the feasibility of using naturally occurring material (Nutraceutical and functional food) that act as probiotics (LF) or prebiotics (TP), to reduce C. albicans counts and biofilm formation,in denture wearers ${ }^{[18,20,52]}$.

Type 1 DS management is based primarily on the adherence to oral/ denture hygiene measures and discontinuous denture wearing ${ }^{[11,54]}$. This treatment protocol was adopted, as the standard treatment,in the current study to be followed by all groups, through-out the experimental period.

(LF) is a commercially available drug that is considered to act as a probiotic. It was chosen for this study due to its' documented effectiveness in improving GIT microbiome, very few adverse reactions, reasonable price, ease of use and availability ${ }^{[28-30]}$. Even though other probiotics may fil these criteria, (LF) was still the drug of choice for this study asin contrast to most probiotics, (LF) comprises heat killed lactobacilli not viable ones. Furthermore, (LF)'s effect on salivary C. albicans, to the best of our knowledge. albicans count and 
biofilm-forming ability have not been reported in literature ${ }^{[29,30]}$. According to the conclusions of the studies performed by Youssef et al. 2019 ${ }^{[30]}$, chronic diarrhea was more markedly improved in the lacteal fort group than in the living lactobacilli group. Moreover, Shripada, Gayatri et al. 2020 ${ }^{[56]}$ Madden and Hunter 2002 ${ }^{[57]}$, stated that heat killed probiotics demonstrate comparable health benefits to live probiotics and a longer shelf life, without carrying any of the latters' potential risks. Whereas Chen, Tsen et al. 2013, stated that heat killed probiotics showedan enhanced immunomodulatory activity in comparison with live strains ${ }^{[27]}$. Heat Killed probiotics are currently referred to as parabiotics ${ }^{[56]}$. The main risks associated with the consumption of live probiotics include, systemic infections due to translocation; interference with gastric colonization in infants; and the acquisition of resistance genes ${ }^{[28]}$.

The prebiotic of choice in this study was a commercially available garlic extract (TP). It imparts the beneficial effects of garlic as a prebiotic without its "in convenient" drawbacks ${ }^{[52]}$. Garlic causes bad breath and body odor, a burning sensation in the oral cavity, heartburn, and nausea, in some instances ${ }^{[36,58]}$. Allicin is the primary bioactive compound in garlic that gives it its` prebiotic quality along with its bad smell, unfortunately ${ }^{[35,36,38]}$. TP is an allicin containing enteric coated tablet that dissolve in the intestine, subsequently alleviating the feeling of discomfort and bad smell, associated with fresh garlic. Several studies demonstrated garlic had promising results in management of

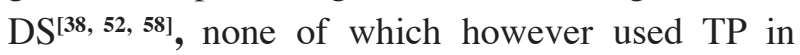
particular. In addition to allicin, TP also contains vitamin $\mathrm{A}, \mathrm{C}$ and $\mathrm{E}$ and zinc sulphate, all of which collectively facilitate tissue regeneration, enhance the immune system and serve as antioxidants ${ }^{[44]}$.

The results of the current work showed that, by the end of the experimental period, Group I (C), revealed a slight reduction in $\mathrm{C}$. albicans counts, compared to base line. This can be attributed to the fact that some of subjects physical condition did not allow them to perform hygiene measures thoroughly or perhaps a longer duration was required for this standard care to have its' full effect Gade, Pawar et al. 2015, stated that for management of DS, oral and denture hygiene must be incessant, for as long as salivary candida counts remain high ${ }^{[11]}$.

Compared to group I (C), groups II (LF) and III (TP) exhibited significantly lower C. albicans counts and biofilm-forming ability at the end of the experimental period. The inflamed erythematous areas associated with DS were also reduced in groups II (LF) and III (TP), again supporting the anti-inflammatory/immunomodulatory effect of both LF and TP.

Despite their different Pharmacodynamics, both LF and TP were able to significantly lower the C. albicans counts and biofilm-forming ability, when compared to the baseline or control group. The results of the current study were consistent with a large body of evidence that supports the role of pre/ probiotics in combating infection ${ }^{[22,31,39,59]}$, immunomodulation ${ }^{[24,29,37,39,43]}$ and improving the overall health of an individual ${ }^{[21]}$. The results of the present work were consistent with those of Silva, M.P. et al. 2016, who stated that heat-killed lactobacilli spp. inhibited germ tube formation of C. albicans ${ }^{[31]}$ and the inhibition of C. albicans biofilms ${ }^{[60]}$. Santos, Jorge et al. 2009, as well reported that probiotics use significantly lowered C. albicans counts in the oral cavity which they attributed to competition between the micro-organisms rather than by stimulation of a specific secretory immune response ${ }^{[61]}$. Ishikawa, Mayer et al. 2015 and Kraft-Bodi, Jørgensen et al. 2015 also reported an resolution of oral candidiasis when patients were treated with probiotics $^{[14,62]}$.

TP was never reported in literature in relevance to DS. However, Khodavandi, Alizadeh et al. 2011, stated that allicin's activity against fungi was comparable to the frequently used antimycotic 
agent fluconazole ${ }^{[42]}$. Bakhshi, Taheri et al. 2012, showed that cconsidering the efficacy of garlic and lack of side effects for this compound and also regarding the nystatin-associated complications, garlic extract can be introduced as a substitution for standard treatment in $\mathrm{DS}^{[52]}$.

Probiotics and prebiotics are believed to surpass conventional treatments in some aspects, as they act as non-specific antimicrobials and thus are very helpful against biofilms, immunomodulators and anti-oxidants with very few reported side-effects.

\section{CONCLUSIONS}

Fromtheresultsof thisstudy, itcanbeconcludedthat 1. Lacteol fort (LF) and Tomex plus (TP) both were effective in reducing $\mathrm{C}$. albicans counts and biofilm-forming ability in denture wearers. 2. LF and TP were able to decrease the inflammatory erythematous areas associated with denture stomatitis (DS).

\section{Recommendation}

Further studies should be carried out for the following:

1. The clinical evaluation of the effect of administration of both Lacteol fort (LF) and Tomex plus (TP) on the oral microbiome.

2. Clinical evaluation of the possible benefits of probiotics and prebiotics treatments in children wearing prosthetic oral appliances.

\section{Declaration of funding}

This research did not receive any specific grant from funding agencies in the public, commercial or not-for-profit sectors.

\section{Conflict of interest}

The Authors declare that there is no conflict of interest.

\section{ACKNOWLEDGMENT}

We would like to thank our colleague Dr. Hala El-Kammar, associate professor of oral pathology, FUE, who offered vision and expertise that greatly helped with the research.

\section{REFERENCES}

1. Cavalheiro, M. and M.C. Teixeira, Candida biofilms: threats, challenges, and promising strategies. Frontiers in medicine, 2018. 5: p. 28.

2. Mayer, F.L., D. Wilson, and B. Hube, Candida albicans pathogenicity mechanisms. Virulence, 2013. 4(2): p.119-128.

3. Sardi, J., et al., Candida species: current epidemiology, pathogenicity, biofilm formation, natural antifungal products and new therapeutic options. Journal of medical microbiology, 2013.62(1): p. 10-24.

4. Coronado-Castellote, L. and Y. Jiménez-Soriano, Clinical and microbiological diagnosis of oral candidiasis. Journal of clinical and experimental dentistry, 2013. 5(5): p. e279.

5. Vylkova, S., et al., The fungal pathogen Candida albicans autoinduces hyphal morphogenesis by raising extracellular pH. MBio, 2011. 2(3): p. e00055-11.

6. Chant, J., Cell polarity in yeast. Annual review of cell and developmental biology, 1999. 15(1): p. 365-391.

7. Tsui, C., E.F. Kong, and M.A. Jabra-Rizk, Pathogenesis of Candida albicans biofilm. FEMS Pathogens and Disease, 2016. 74(4): p. ftw018.

8. Uppuluri, P., et al., Candida albicans dispersed cells are developmentally distinct from biofilm and planktonic cells. MBio, 2018. 9(4): p. e01338-18.

9. Nevzatoğlu, E.U., et al., Adherence of Candida albicans to denture base acrylics and silicone-based resilient liner materials with different surface finishes. Clinical oral investigations, 2007. 11(3): p. 231-236.

10. Martins, K.V. and S.M. de Lacerda Gontijo, Treatment of denture stomatitis: literature review. Rev Bras Odontol, 2017. 74(3): p. 215-220.

11. Gade, J., D. Pawar, and N. Singh, Review on Denture Stomatitis: Classification, clinical features and treatment. IOSR J. Dent. Med. Sci. Ver, 2015. 14(12): p. 2279-861.

12. Sicard, L., et al., Orthodontics and oral mucosal lesions in children and teenagers. Journal of Dentofacial Anomalies and Orthodontics, 2018. 21(2): p. 207. 
13. Uzunoglu, E., et al., Biofilm-forming ability and adherence to poly-(methyl-methacrylate) acrylic resin materials of oral Candida albicans strains isolated from HIV positive subjects. The journal of advanced prosthodontics, 2014. 6(1): p. 30-34.

14. Ishikawa, K.H., et al., A multispecies probiotic reduces oral Candida colonization in denture wearers. Journal of Prosthodontics, 2015. 24(3): p. 194-199.

15. Coco, B., et al., Mixed Candida albicans and Candida glabrata populations associated with the pathogenesis of denture stomatitis. Oral microbiology and immunology, 2008. 23(5): p. 377-383.

16. Pelletier, R., et al., Emergence of disseminated candidiasis caused by Candida krusei during treatment with caspofungin: case report and review of literature. Medical mycology, 2005. 43(6): p. 559-564.

17. Ksiezopolska, E. and T. Gabaldón, Evolutionary emergence of drug resistance in Candida opportunistic pathogens. Genes, 2018. 9(9): p. 461.

18. Pappas, P.G., et al., Clinical practice guideline for the management of candidiasis: 2016 update by the Infectious Diseases Society of America. Clinical Infectious Diseases, 2015. 62(4): p. e1-e50.

19. Lyu, X., et al., Efficacy of nystatin for the treatment of oral candidiasis: a systematic review and meta-analysis. Drug design, development and therapy, 2016. 10: p. 1161.

20. Mishra, S.S., et al., Advances in probiotics, prebiotics and nutraceuticals, in Innovations in Technologies for Fermented Food and Beverage Industries. 2018, Springer. p. 121-141.

21. Maftei, N.-M., Probiotic, Prebiotic and Synbiotic Products in Human Health, in Frontiers and New Trends in the Science of Fermented Food and Beverages. 2019, IntechOpen.

22. Roberfroid, M., Prebiotics: the concept revisited. The Journal of nutrition, 2007. 137(3): p. 830S-837S.

23. Aronson, J.K., Defining 'nutraceuticals': neither nutritious nor pharmaceutical. British journal of clinical pharmacology, 2017. 83(1): p. 8-19.

24. Bermudez-Brito, M., et al., Probiotic mechanisms of action. Annals of Nutrition and Metabolism, 2012. 61(2): p. 160-174.

25. Mendonça, F.H.B.P., et al., Effects of probiotic bacteria on Candida presence and IgA anti-Candida in the oral cavity of elderly. Brazilian dental journal, 2012. 23(5): p. 534-538.
26. Thomas, C.M. and J. Versalovic, Probiotics-host communication: Modulation of signaling pathways in the intestine. Gut microbes, 2010. 1(3): p. 148-163.

27. Chen, C.-Y., et al., Enhancement of the immune response against Salmonella infection of mice by heat-killed multispecies combinations of lactic acid bacteria. Journal of medical microbiology, 2013. 62(11): p. 1657-1664.

28. Piqué, N., M. Berlanga, and D. Miñana-Galbis, Health benefits of heat-killed (Tyndallized) probiotics: an overview. International journal of molecular sciences, 2019. 20(10): p. 2534.

29. Xiao, S. D., et al., Multicenter, randomized, controlled trial of heat-killedLactobacillus acidophilus LB in patients with chronic diarrhea. Advances in therapy, 2003. 20(5): p. 253-260.

30. Youssef, D.M., et al., Effect of Combined Heat killed Probiotic and Wheat Bran on Gastrointestinal Symptoms in Hemodialysis Children. GEGET, 2019. 14(1): p. 8-14.

31. Silva, M.P., et al., Probiotics for Prevention and Treatment of Candidiasis and Other Infectious Diseases: Lactobacillus spp. and Other Potential Bacterial Species. Probiotics and Prebiotics in Human Nutrition and Health, 2016. 13: p. 241.

32. Andresen, V., et al., Efficacy of freeze-dried Lactobacilli in functional diarrhoe: a pilot study. Deutsche medizinische Wochenschrift (1946), 2012. 137(37): p. 1792-1796.

33. Lievin-Le Moal, V., et al., Lactobacillus acidophilus (strain LB) from the resident adult human gastrointestinal microflora exerts activity against brush border damage promoted by a diarrhoeagenic Escherichia coli in human enterocyte-like cells. Gut, 2002. 50(6): p. 803-811.

34. Hatakka, K., et al., Probiotics reduce the prevalence of oral Candida in the elderly - a randomized controlled trial. Journal of dental research, 2007. 86(2): p. 125-130.

35. Müller, A., et al., Allicin induces thiol stress in bacteria through S-allylmercapto modification of protein cysteines. Journal of Biological Chemistry, 2016. 291(22): p. 11477-11490.

36. Farías-Campomanes, A.M., et al., Allicin-rich extract obtained from garlic by pressurized liquid extraction: quantitative determination of allicin in garlic samples. Food and Public Health, 2014. 4(6): p. 272-278.

37. Nakamoto, M., et al., Antimicrobial properties of hydrophobic compounds in garlic: Allicin, vinyldithiin, ajoene and diallyl polysu Lacteol fort (LF) ides. Experimental and Therapeutic Medicine, 2020. 
38. Si, X.-B., et al., Allicin as add-on therapy for Helicobacter pylori infection: A systematic review and meta-analysis. World journal of gastroenterology, 2019. 25(39): p. 6025.

39. Davis, S.R., An overview of the antifungal properties of allicin and its breakdown products-the possibility of a safe and effective antifungal prophylactic. Mycoses, 2005. 48(2): p. 95-100.

40. Borlinghaus, J., et al., Allicin: chemistry and biological properties. Molecules, 2014. 19(8): p. 12591-12618.

41. Salehi, B., et al., Allicin and health: A comprehensive review. Trends in food science \& technology, 2019.

42. Khodavandi, A., et al., Comparison between efficacy of allicin and fluconazole against Candida albicans in vitro and in a systemic candidiasis mouse model. FEMS microbiology letters, 2011.315(2): p. 87-93.

43. Yu, L., et al., Allicin-induced global gene expression profile of Saccharomyces cerevisiae. Applied microbiology and biotechnology, 2010. 88(1): p. 219-229.

44. Gombart, A.F., A. Pierre, and S. Maggini, A Review of Micronutrients and the Immune System-Working in Harmony to Reduce the Risk of Infection. Nutrients, 2020. 12(1): p. 236.

45. Pattanaik, S., et al., Denture Stomatitis: A literature review. Journal of Indian Academy of Oral Medicine and Radiology, 2010.22(3): p. 136.

46. Rickham, P., Human experimentation. Code of ethics of the world medical association. Declaration of Helsinki. British medical journal, 1964. 2(5402): p. 177-177.

47. Lombardi, T. and E. Budtz-Jørgensen, Treatment of dentureinduced stomatitis: a review. The European journal of prosthodontics and restorative dentistry, 1993. 2(1): p. 17-22.

48. Farah, C., N. Lynch, and M. McCullough, Oral fungal infections: an update for the general practitioner. Australian dental journal, 2010. 55: p. 48-54.

49. Henson, B.S. and D.T. Wong, Collection, storage, and processing of saliva samples for downstream molecular applications, in Oral Biology. 2010, Springer. p. 21-30.

50. Falsafi, P., et al., Salivary Transferrin Levels in Patients with Oral Lichen Planus. Pesquisa Brasileira em Odontopediatria e Clínica Integrada, 2019. 19.
51. Yu, W., K.M. Hallinen, and K.B. Wood, Interplay between antibiotic efficacy and drug-induced lysis underlies enhanced biofilm formation at subinhibitory drug concentrations. Antimicrobial agents and chemotherapy, 2018. 62(1): p. e01603-17.

52. Bakhshi, M., et al., Comparison of therapeutic effect of aqueous extract of garlic and nystatin mouthwash in denture stomatitis. Gerodontology, 2012. 29(2): p. e680-e684.

53. Scheibler, E., et al., Use of nystatin and chlorhexidine in oral medicine: Properties, indications and pitfalls with focus on geriatric patients. Gerodontology, 2017. 34(3): p. 291-298.

54. Walsh, T., P. Riley, and A. Veitz-Keenan, Interventions for managing denture stomatitis. Cochrane Database of Systematic Reviews, 2015(10).

55. Dismukes, W.E., Introduction to antifungal drugs. Clinical Infectious Diseases, 2000: p. 653-657.

56. Shripada, R., A.-J. Gayatri, and P. Sanjay, Paraprobiotics. Precision Medicine for Investigators, Practitioners and Providers, 2020: p. 39-49.

57. Madden, J. and J. Hunter, A review of the role of the gut microflora in irritable bowel syndrome and the effects of probiotics. British Journal of Nutrition, 2002. 88(S1): p. s67-s72.

58. Shuford, J.A., J.M. Steckelberg, and R. Patel, Effects of fresh garlic extract on Candida albicans biofilms. Antimicrobial agents and chemotherapy, 2005. 49(1): p. 473-473.

59. Kim, Y.-S., et al., Quantitative and qualitative analysis of the antifungal activity of allicin alone and in combination with antifungal drugs. PLoS One, 2012.7(6).

60. Rossoni, R.D., et al., Antifungal activity of clinical Lactobacillus strains against Candida albicans biofilms: identification of potential probiotic candidates to prevent oral candidiasis. Biofouling, 2018. 34(2): p. 212-225.

61. Santos, A.L.d., et al., Influence of probiotics on Candida presence and IgA anti-Candida in the oral cavity. Brazilian Journal of Microbiology, 2009. 40(4): p. 960-964.

62. Kraft-Bodi, E., et al., Effect of probiotic bacteria on oral Candida in frail elderly. Journal of dental research, 2015. 94(9_suppl): p. 181S-186S. 